\title{
The Alpha-Beta Skew Logistic Distribution: Properties and Applications
}

\author{
Hamid Esmaeili, Fazlollah Lak*, Morad Alizadeh, Mohammad Esmail Dehghan Monfared \\ Department of Statistics, Persian Gulf University, Bushehr, Iran
}

\begin{abstract}
A new family of skew distributions is introduced by extending the alpha skew logistic distribution proposed by Hazarika-Chakraborty [9]. This family of distributions is called the alpha-beta skew logistic (ABSLG) distribution. Density function, moments, skewness and kurtosis coefficients are derived. The parameters of the new family are estimated by maximum likelihood and moments methods. The performance of the obtained estimators examined via a Monte carlo simulation. Flexibility, usefulness and suitability of ABSLG is illustrated by analyzing two real data sets.
\end{abstract}

Keywords Logistic distribution, Maximum likelihood, Moments method, Skew distribution.

AMS 2010 subject classifications 60E05, 62F10, 62G05

DOI: $10.19139 /$ soic-2310-5070-706

\section{Introduction}

Let the random variable $\mathrm{X}$ have the logistic distribution with probability density function (pdf),

$$
f(x ; \mu, \sigma)=\frac{e^{-\left\{\frac{x-\mu}{\sigma}\right\}}}{\sigma\left(1+e^{-\left\{\frac{x-\mu}{\sigma}\right\}}\right)^{2}}, \quad x, \mu \in R, \sigma>0,
$$

which is denoted by $X \sim L G(\mu, \sigma)$. [10], proposed the general formula for the construction of skew-symmetric distributions starting from a symmetric (about 0 ) pdf $h(\cdot)$ and a skew function $G(\cdot)$, a Lebesgue measurable function such that, $0 \leq G(z) \leq 1$ and $G(z)+G(-z)=1, Z \in R$ almost everywhere. A random variable $\mathrm{Z}$ is said to be skew symmetric if its pdf is of the following form:

$$
f_{Z}(z)=2 h(z) G(z), \quad z \in R .
$$

Choosing an appropriate skew function $G(z)$ in the equation (1), one can construct different skew distributions with both unimodal and multimodal behavior. $[4,5,6]$ introduced the new skew logistic, the skew Laplace and the skew normal distributions. [2] proposed the skew normal (SN) distribution with the pdf $\phi_{S N}(x ; \alpha)=2 \phi(x) \Phi(\alpha x)$ for $(\alpha, x) \in R^{2}$. Elal-olivero introduced an alpha skew normal(ASN) and [12] extended the ASN to alpha-beta skew normal distribution.

Recently, [9] introduced a new class of skew logistic distributions called alpha skew logistic(ASLG) distributions, with the pdf,

$$
f_{Z}(z ; \alpha)=\frac{3\left((1-\alpha z)^{2}+1\right)}{\left(6+\alpha^{2} \pi^{2}\right)} \times \frac{e^{-z}}{\left(1+e^{-z}\right)^{2}}, \quad(\alpha, z)^{T} \in R^{2}
$$

\footnotetext{
*Correspondence to: Fazlollah Lak (Email: fazlollahlak@ gmail.com). Department of Statistics, Persian Gulf University, Bushehr, Iran.
}

ISSN 2310-5070 (online) ISSN 2311-004X (print)

Copyright (C) 2020 International Academic Press 
The aim of this paper is to introduce a new family of distributions as an extension of ASLG distribution with the pdf (3). In the context of distribution theory, we can refer to recent articles by Mozafari et al. [11] and Alizadeh et al. [1]. The rest of this paper is organized as follows: In Section 2, the new family of distributions is defined and studied in details(pdf, cdf, moments).In Section 3, truncated function has been investigated.In Section 4,method of moments estimates(MMEs) and maximum likelihood estimates(MLEs) are derived. In Section 5, simulation is performed using Monte Carlo method. In Section 6, to illustrate the applicability of the proposed model, two real data sets are analysed.

\section{The Family of Alpha-Beta SLG Distribution}

In this Section, the new family is introduced. This new family of skew distributions with more flexibility than the Hazarikas ASLG is introduced. The random variable $Z$ follows the alpha-beta SLG distributions, denoted by $\operatorname{ABSLG}(\alpha, \beta)$, if it has the pdf,

$$
f(z ; \alpha, \beta)=\frac{\left(1-\alpha z-\beta z^{3}\right)^{2}+1}{2+\alpha^{2} \frac{\pi^{2}}{3}+\frac{31}{21} \beta^{2} \pi^{6}+\frac{14}{15} \alpha \beta \pi^{4}} \times \frac{e^{-z}}{\left(1+e^{-z}\right)^{2}} .
$$

The function of (4) for given $\alpha$ and $\beta$ is a proper pdf since $f(z ; \alpha, \beta) \geq 0$ for all $(z, \alpha, \beta)^{T} \in R^{3}$, and

$$
\int_{-\infty}^{\infty} f(z ; \alpha, \beta) d z=1
$$

The equation (5) is obtained because,

$$
\int_{-\infty}^{\infty}\left[\left(1-\alpha z-\beta z^{3}\right)^{2}+1\right] \times \frac{e^{-z}}{\left(1+e^{-z}\right)^{2}} d z=2+\alpha^{2} \frac{\pi^{2}}{3}+\frac{31}{21} \beta^{2} \pi^{6}+\frac{14}{15} \alpha \beta \pi^{4} .
$$

The above integral can be obtained by the moments of the standard logistic distribution. Figure 1 presents the ABSLG pdf for different choices of the parameters $\alpha$ and $\beta$. It can be seen from Figure 1 that the proposed model has at most four modes, also the effects on the skewness can be seen.

Some properties of the $\operatorname{ABSLG}(\alpha, \beta)$ distribution are as follow:

- For $\alpha=\beta=0$, we get the standard logistic distribution which is given by

$$
f(z)=\frac{e^{-z}}{\left(1+e^{-z}\right)^{2}}, \quad z \in R,
$$

and denoted by $Z \sim L G(0,1)$.

- For $\beta=0$ the pdf (4) is simplified as the pdf of the ASLG distribution.

- For $\alpha=0$, the pdf (4) is simplified as the following pdf,

$$
f(z ; \beta)=\frac{\left(1-\beta z^{3}\right)^{2}+1}{2+\frac{31}{21} \beta^{2} \pi^{6}} \times \frac{e^{-z}}{\left(1+e^{-z}\right)^{2}}, \quad z \in R .
$$

The above equation is referred to as the beta skew logistic(BSLG) distribution.

- If $\alpha \longrightarrow \pm \infty$, then we get a bimodal logistic (BLG) distribution given by,

$$
f(z)=\frac{3 z^{2} e^{-z}}{\pi^{2}\left(1+e^{-z}\right)^{2}}, \quad z \in R .
$$

- If $Z \sim A B S L G(\alpha, \beta)$, then $-Z \sim A B S L G(-\alpha,-\beta)$. 


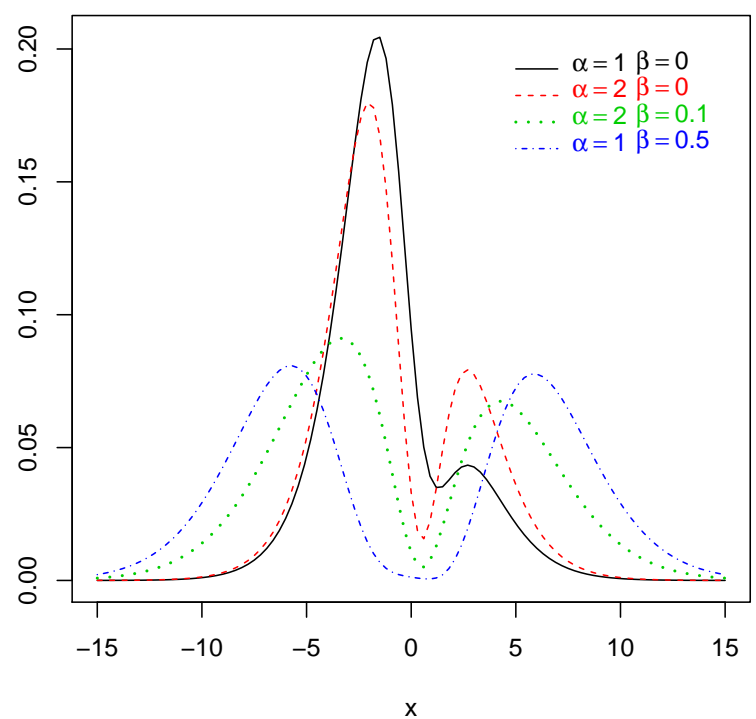

Figure 1. The pdf of the $\operatorname{ABSLG}(\alpha, \beta)$ distribution for some selected values of the parameters.

Here, we prove that the ABSLG has at most four modes. The first derivative of the pdf $f(z ; \alpha, \beta)$ of $\operatorname{ABSLG}(\alpha, \beta)$ with respect to $\mathrm{z}$ is given by,

$$
\begin{aligned}
\frac{\partial f(z ; \alpha, \beta)}{\partial z} & =-\left(\left(-\beta z^{3}-\alpha z+1\right)^{2}+1\right) \frac{e^{-z}}{\left(1+e^{-z}\right)^{2}} \\
& +2\left(-\beta z^{3}-\alpha z+1\right)\left(-\beta^{2} z^{2}-\alpha\right) \frac{e^{-z}}{\left(1+e^{-z}\right)^{2}} \\
& +2\left(\left(-\beta z^{3}-\alpha z+1\right)^{2}+1\right) \frac{e^{-2 z}}{\left(1+e^{-z}\right)^{2}}=0
\end{aligned}
$$

This expression has at most seven zeros. Thus, the function $f(z ; \alpha, \beta)$ can have at most four modes.

\subsection{Cumulative Distribution Function(CDF)}

The cdf of the $\operatorname{ABSLG}(\alpha, \beta)$ is obtained by,

$$
F_{Z}(z ; \alpha, \beta)=\frac{1}{c(\alpha, \beta)} \int_{-\infty}^{z}\left(\left(1-\alpha z-\beta z^{3}\right)^{2}+1\right) \times \frac{e^{-z}}{\left(1+e^{-z}\right)^{2}} d z
$$


such that,

$$
\begin{aligned}
\int((1 & \left.\left.-\alpha z-\beta z^{3}\right)^{2}+1\right) \frac{e^{-z}}{\left(1+e^{-z}\right)^{2}} d z \\
& =-2 \alpha(\alpha z-1)\left(\log \left(e^{z}+1\right)-z\right) \\
& -2 \beta z^{2}\left(z\left(3 \beta z^{2}+4 \alpha\right)-3\right) \log \left(e^{z}+1\right)-720 \beta^{2} L i_{6}\left(-e^{z}\right) \\
& +720 \beta^{2} z L i_{5}\left(-e^{z}\right)-24 \beta\left(15 \beta z^{2}+2 \alpha\right) L i_{4}\left(-e^{z}\right) \\
& +12 \beta\left(2 z\left(5 \beta z^{2}+2 \alpha\right)-1\right) L i_{3}\left(-e^{z}\right) \\
& -6 \beta z\left(z\left(5 \beta z^{2}+4 \alpha\right)-2\right) L i_{2}\left(-e^{z}\right)+2 \alpha^{2} L i_{2}\left(-e^{-z}\right) \\
& +\frac{\beta z^{3}\left(z\left(\beta z^{2}+2 \alpha\right)-2\right) e^{z}-\alpha^{2} z^{2}+2 \alpha z-2}{e^{z}+1},
\end{aligned}
$$

where $c(\alpha, \beta)=2+\alpha^{2} \frac{\pi^{2}}{3}+\frac{31}{21} \beta^{2} \pi^{6}+\frac{14}{15} \alpha \beta \pi^{4}$ and $L i_{n}(z)=\sum_{k=1}^{\infty} \frac{Z^{k}}{k^{n}}$ is the poly-logarithm function.

\subsection{Moments}

The mean and moments of distributions are usually used as measures of central indices in a population. In the following theorem, the moments of proposed distribution is investigated.

Theorem 2.1. The $k^{\text {th }}$ order moment of $A B S L G(\alpha, \beta)$ distribution is given by,

$$
E\left(Z^{k}\right)=\frac{1}{2+\alpha^{2} \frac{\pi^{2}}{3}+\frac{31}{21} \beta^{2} \pi^{6}+\frac{14}{15} \alpha \beta \pi^{4}}= \begin{cases}A, & \text { if } k \text { is even }, \\ B, & \text { if } k \text { is odded }\end{cases}
$$

Such that,

$$
\begin{aligned}
A=2\left(1-\frac{1}{2^{k-1}}\right) \Gamma(k+1) \zeta(k) & +\alpha^{2}\left(1-\frac{1}{2^{k+1}}\right) \Gamma(k+3) \zeta(k+2) \\
& +\beta^{2}\left(1-\frac{1}{2^{k+5}}\right) \Gamma(k+7) \zeta(k+6) \\
& +2 \alpha \beta\left(1-\frac{1}{2^{k+3}}\right) \Gamma(k+5) \zeta(k+4),
\end{aligned}
$$

and

$$
B=-2 \alpha\left(1-\frac{1}{2^{k}}\right) \Gamma(k+2) \zeta(k+1)-2 \beta\left(1-\frac{1}{2^{k+2}}\right) \Gamma(k+4) \zeta(k+3),
$$

where $\zeta(s)=\sum_{j}^{\infty} j^{-s}$ is the riemann zeta functions and $\Gamma(s)=\int_{0}^{\infty} t^{s-1} e^{-t} d t$.

Proof

Recall that, for $X \sim L G(0,1)$ i.e. for the standard logistic random variable $\mathrm{X}$,

$$
E\left(X^{k}\right)=\left\{\begin{array}{cl}
2\left(1-\frac{1}{2^{k-1}}\right) \Gamma(k+1) \zeta(k), & \text { if } \mathrm{k} \text { is even } \\
0, & \text { if } \mathrm{k} \text { is odded }
\end{array}\right.
$$


Now,

$$
\begin{aligned}
E\left(Z^{k}\right) & =\int_{-\infty}^{+\infty} z^{k} f(z ; \alpha, \beta) d z \\
& =\frac{1}{C(\alpha, \beta)} \int_{-\infty}^{+\infty} z^{k}\left(2-2 \alpha z+\alpha^{2} z^{2}\right. \\
& \left.+\beta^{2} z^{6}+2 \alpha \beta z^{4}-2 \beta z^{3}\right) \frac{e^{-z}}{\left(1+e^{-z}\right)^{2}} d z \\
& =\frac{1}{C(\alpha, \beta)}\left(\int_{-\infty}^{+\infty} 2 z^{k} \frac{e^{-z}}{\left(1+e^{-z}\right)^{2}} d z-\int_{-\infty}^{+\infty} 2 \alpha z^{k+1} \frac{e^{-z}}{\left(1+e^{-z}\right)^{2}} d z\right. \\
& +\int_{-\infty}^{+\infty} \alpha^{2} z^{k+2} \frac{e^{-z}}{\left(1+e^{-z}\right)^{2}} d z+\int_{-\infty}^{+\infty} \beta^{2} z^{k+6} \frac{e^{-z}}{\left(1+e^{-z}\right)^{2}} d z \\
& \left.+\int_{-\infty}^{+\infty} 2 \alpha \beta z^{k+4} \frac{e^{-z}}{\left(1+e^{-z}\right)^{2}} d z-\int_{-\infty}^{+\infty} 2 \beta z^{k+3} \frac{e^{-z}}{\left(1+e^{-z}\right)^{2}} d z\right)
\end{aligned}
$$

where $C(\alpha, \beta)=2+\alpha^{2} \frac{\pi^{2}}{3}+\frac{31}{21} \beta^{2} \pi^{6}+\frac{14}{15} \alpha \beta \pi^{4}$.

Case1. When $k$ is even then,

$$
\begin{aligned}
E\left(Z^{k}\right) & =\frac{1}{C(\alpha, \beta)}\left(\int_{-\infty}^{+\infty} 2 z^{k} \frac{e^{-z}}{\left(1+e^{-z}\right)^{2}} d z+\int_{-\infty}^{+\infty} \alpha^{2} z^{k+2} \frac{e^{-z}}{\left(1+e^{-z}\right)^{2}} d z\right. \\
& \left.+\int_{-\infty}^{+\infty} \beta^{2} z^{k+6} \frac{e^{-z}}{\left(1+e^{-z}\right)^{2}} d z+\int_{-\infty}^{+\infty} 2 \alpha \beta z^{k+4} \frac{e^{-z}}{\left(1+e^{-z}\right)^{2}} d z\right) \\
& =\frac{1}{C(\alpha, \beta)}\left(2\left(1-\frac{1}{2^{k-1}}\right) \Gamma(k+1) \zeta(k)\right. \\
& +\alpha^{2}\left(1-\frac{1}{2^{k+1}}\right) \Gamma(k+3) \zeta(k+2) \\
& +\beta^{2}\left(1-\frac{1}{2^{k+5}}\right) \Gamma(k+7) \zeta(k+6) \\
& \left.+2 \alpha \beta\left(1-\frac{1}{2^{k+3}}\right) \Gamma(k+5) \zeta(k+4)\right)
\end{aligned}
$$

where $C(\alpha, \beta)=2+\alpha^{2} \frac{\pi^{2}}{3}+\frac{31}{21} \beta^{2} \pi^{6}+\frac{14}{15} \alpha \beta \pi^{4}$.

\section{Case2. When $k$ is odd then}

$$
\begin{aligned}
E\left(Z^{k}\right) & =\frac{1}{C(\alpha, \beta)}\left(-\int_{-\infty}^{+\infty} 2 \alpha z^{k+1} \frac{e^{-z}}{\left(1+e^{-z}\right)^{2}} d z\right. \\
& \left.-\int_{-\infty}^{+\infty} 2 \beta z^{k+3} \frac{e^{-z}}{\left(1+e^{-z}\right)^{2}} d z\right) \\
& =-2 \alpha\left(1-\frac{1}{2^{k}}\right) \Gamma(k+2) \zeta(k+1) \\
& -2 \beta\left(1-\frac{1}{2^{k+2}}\right) \Gamma(k+4) \zeta(k+3) .
\end{aligned}
$$

The proof is completed.

From Theorem 2.1, using the following particular values of the Riemann zeta functions,

$$
\zeta(2)=\frac{\pi^{2}}{6}, \zeta(4)=\frac{\pi^{4}}{90} \text { and } \zeta(6)=\frac{\pi^{6}}{945} .
$$


We obtain,

$$
E(Z)=\mu_{1}^{\prime}=\frac{1}{2+\alpha^{2} \frac{\pi^{2}}{3}+\frac{31}{21} \beta^{2} \pi^{6}+\frac{14}{15} \alpha \beta \pi^{4}}\left(\frac{-5 \alpha \pi^{2}-\beta \pi^{4}}{15}\right) .
$$

It is proportional to variance of standard logistic distribution and positive (or negative) if $-5 \alpha \pi^{2}-\beta \pi^{4}<(>) 0$.

$$
\begin{aligned}
& E\left(Z^{2}\right)=\mu_{2}^{\prime}=\frac{1}{C(\alpha, \beta)}\left(\frac{70 \pi^{2}+7 \alpha^{2} \pi^{4}+465 \alpha \beta \pi^{6}+896 \beta^{2} \pi^{8}}{210}\right) \\
& E\left(Z^{3}\right)=\mu_{3}^{\prime}=\frac{1}{C(\alpha, \beta)}\left(\frac{-49 \alpha \pi^{4}-155 \beta \pi^{6}}{105}\right) \\
& E\left(Z^{4}\right)=\mu_{4}^{\prime}=\frac{1}{C(\alpha, \beta)}\left(\frac{7}{15} \pi^{4}+\frac{31}{42} \alpha^{2} \pi^{6}+\frac{128}{15} \alpha \beta \pi^{8}+\frac{2555}{66} \beta^{2} \pi^{10}\right),
\end{aligned}
$$

and the variance of $A B S L G(\alpha, \beta)$ is obtained by,

$$
\begin{aligned}
\operatorname{var}(Z) & =E\left(Z^{2}\right)-E^{2}(Z) \\
& =\frac{1}{C(\alpha, \beta)}\left(\frac{70 \pi^{2}+7 \alpha^{2} \pi^{4}+465 \alpha \beta \pi^{6}+896 \beta^{2} \pi^{8}}{210}\right) \\
& -\left(\frac{1}{C(\alpha, \beta)} \frac{-5 \alpha \pi^{2}-\beta \pi^{4}}{15}\right)^{2},
\end{aligned}
$$

\subsection{Skewness and Kurtosis}

A fundamental task in many statistical analyses is to characterize the location and variability of a data set. A further characterization of the data includes skewness and kurtosis. The skewness and kurtosis of proposed distribution is investigated in the following theorem.

Theorem 2.2. Let $Z \sim A B S L G(\alpha, \beta)$, then $\gamma_{1} \in(-2.13,2.13)$ and $\gamma_{2} \in(-1.24,3.96)$ where $\gamma_{1}$ and $\gamma_{2}$ stand for the skewness and kurtosis coefficients of the distribution of $Z$.

Proof

By the moments and Theorem 2.1,

$$
\gamma_{1}=\frac{E\left(Z^{3}\right)-3 E(Z) E\left(Z^{2}\right)+2 E^{3}(Z)}{\left(E\left(Z^{2}\right)-E^{2}(Z)\right)^{\frac{3}{2}}}=\frac{A}{B^{\frac{3}{2}}},
$$

where,

$$
\begin{aligned}
& A=\left(\frac{1}{C(\alpha, \beta)}\left(\frac{-49 \alpha \pi^{4}-155 \beta \pi^{6}}{105}\right)\right. \\
& -3\left(\frac{1}{C(\alpha, \beta)}\right)^{2}\left(\frac{-5 \alpha \pi^{2}-\beta \pi^{4}}{15}\right)\left(\frac{70 \pi^{2}+7 \alpha^{2} \pi^{4}+465 \alpha \beta \pi^{6}+896 \beta^{2} \pi^{8}}{210}\right) \\
& \left.+2\left(\frac{1}{C(\alpha, \beta)}\left(\frac{-5 \alpha \pi^{2}-\beta \pi^{4}}{15}\right)\right)^{3}\right),
\end{aligned}
$$

and,

$$
\begin{aligned}
B= & \frac{1}{C(\alpha, \beta)}\left(\frac{70 \pi^{2}+7 \alpha^{2} \pi^{4}+465 \alpha \beta \pi^{6}+896 \beta^{2} \pi^{8}}{210}\right) \\
& -\left(\frac{1}{C(\alpha, \beta)}\left(\frac{-5 \alpha \pi^{2}-\beta \pi^{4}}{15}\right)\right)^{2} .
\end{aligned}
$$


And,

$$
\begin{aligned}
\gamma_{2} & =\frac{E\left(Z^{4}\right)-4 E(Z) E\left(Z^{3}\right)+6 E^{2}(Z) E\left(Z^{2}\right)-3 E^{4}(Z)}{\left(E\left(Z^{2}\right)-E^{2}(Z)\right)^{2}}-3 \\
& =-3+\frac{\frac{1}{C(\alpha, \beta)}\left(\frac{7}{15} \pi^{4}+\frac{31}{42} \alpha^{2} \pi^{6}+\frac{128}{15} \alpha \beta \pi^{8}+\frac{2555}{66} \beta^{2} \pi^{10}\right)}{D} \\
& -\frac{4\left(\frac{1}{C(\alpha, \beta)}\right)^{2}\left(\frac{-5 \alpha \pi^{2}-\beta \pi^{4}}{15}\right)\left(\frac{-49 \alpha \pi^{4}-155 \beta \pi^{6}}{105}\right)}{D} \\
& +\frac{6\left(\frac{1}{C(\alpha, \beta)}\right)^{3}\left(\frac{-5 \alpha \pi^{2}-\beta \pi^{4}}{15}\right)^{2}\left(\frac{70 \pi^{2}+7 \alpha^{2} \pi^{4}+465 \alpha \beta \pi^{6}+896 \beta^{2} \pi^{8}}{210}\right)}{D} \\
& -\frac{3\left(\frac{1}{C(\alpha, \beta)}\left(\frac{-5 \alpha \pi^{2}-\beta \pi^{4}}{15}\right)\right)^{4}}{D},
\end{aligned}
$$

such that,

$$
\begin{aligned}
D & =\left(\frac{1}{C(\alpha, \beta)}\left(\frac{70 \pi^{2}+7 \alpha^{2} \pi^{4}+465 \alpha \beta \pi^{6}+896 \beta^{2} \pi^{8}}{210}\right)\right. \\
& \left.-\left(\frac{1}{C(\alpha, \beta)}\left(\frac{-5 \alpha \pi^{2}-\beta \pi^{4}}{15}\right)\right)^{2}\right)^{2} .
\end{aligned}
$$

By using a numerical method the desired results follow.
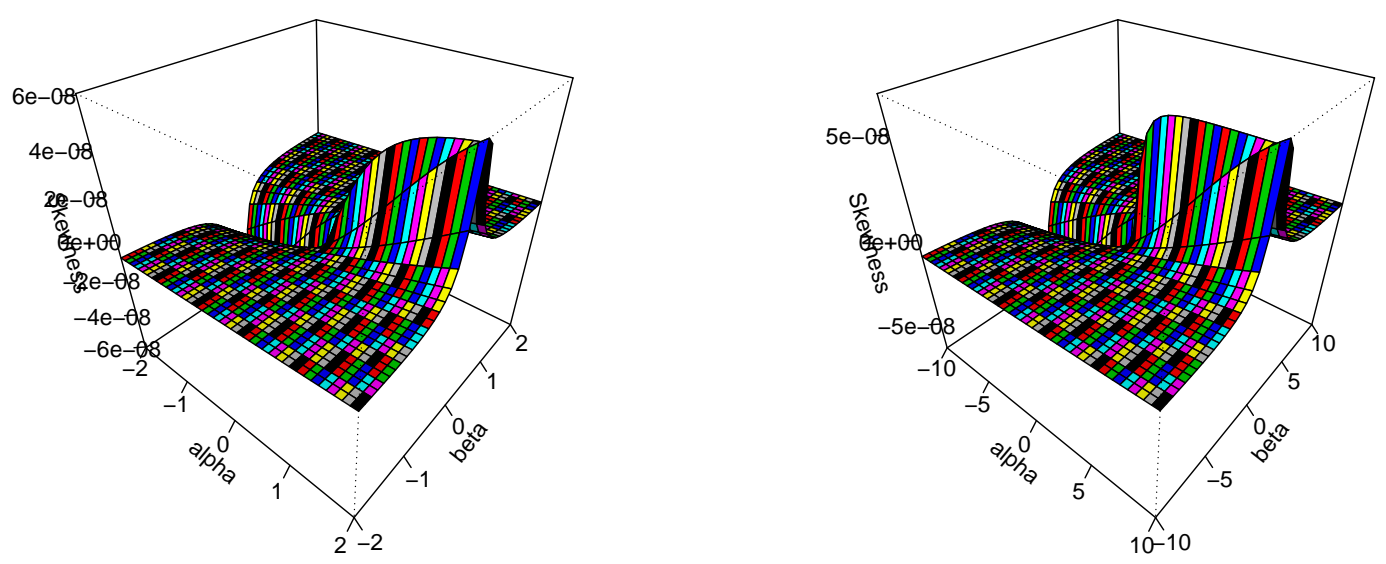

Figure 2. The skewness function of the $\operatorname{ABSLG}(\alpha, \beta)$ distribution. 


\section{Truncated ABSLG $(\alpha, \beta)$}

In this section alpha-beta skew logistic distribution truncated below 0 is presented as a potential life time distribution. The pdf of alpha-beta skew logistic distribution which is truncated below 0 is denoted as $\operatorname{TABSLG}(\alpha, \beta)$ and is given by,

$$
\begin{aligned}
f_{T}(t ; \alpha, \beta)= & \frac{\left(1-\alpha t-\beta t^{3}\right)^{2}+1}{\frac{1}{2}+\alpha^{2} \frac{\pi^{2}}{6}+\frac{31}{42} \beta^{2} \pi^{6}+\frac{7}{15} \alpha \beta \pi^{4}-1.38 \alpha+10.82 \beta} \\
& \times \frac{e^{-t}}{\left(1-e^{-t}\right)^{2}}, \quad t>0,
\end{aligned}
$$

Where,

$$
\frac{1}{2}+\alpha^{2} \frac{\pi^{2}}{6}+\frac{31}{42} \beta^{2} \pi^{6}+\frac{7}{15} \alpha \beta \pi^{4}-1.38 \alpha+10.82 \beta>0 .
$$

The survival function $S_{T}(t ; \alpha, \beta)$ and the hazard function $h_{T}(t ; \alpha, \beta)$ of $T A B S L G(\alpha, \beta)$ can be easily expressed in terms of the pdf $f_{T}(t ; \alpha, \beta)$ and $\operatorname{cdf} F_{T}(t ; \alpha, \beta)$ of $T A B S L G(\alpha, \beta)$ as,

$$
S_{T}(t ; \alpha, \beta)=\frac{1-F_{T}(t ; \alpha, \beta)}{1-F_{T}(0 ; \alpha, \beta)},
$$

and,

$$
h_{T}(t ; \alpha, \beta)=\frac{f_{T}(t ; \alpha, \beta)}{S_{T}(t ; \alpha, \beta)}=\frac{f_{T}(t ; \alpha, \beta)}{1-F_{T}(t ; \alpha, \beta)} .
$$

Remark 3.1. Clearly, for $\alpha, \beta=0, T A B S L G(\alpha, \beta)$ is simplified to the standard half logistic distribution, [3].

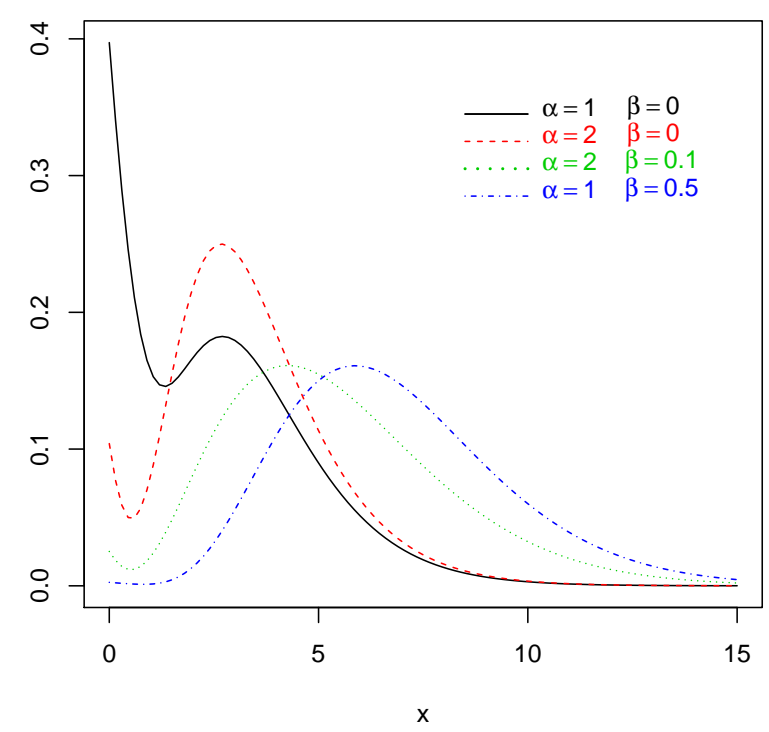

Figure 3. The hazard function of the $\operatorname{ABSLG}(\alpha, \beta)$ distribution for some selected values of the parameters. 


\section{Parameter Estimation}

This section is denoted to the estimation of parameters of $A B S L G(\alpha, \beta)$. If $Z \sim A B S L G(\alpha, \beta)$, then $Y=$ $\mu+\sigma Z$ is said to be the location $(\mu)$ and scale extension $(\sigma)$ of $Z$ and has the pdf,

$$
f_{Z}(z ; \alpha, \beta, \mu, \sigma)=\frac{\left(1-\alpha\left(\frac{y-\mu}{\sigma}\right)-\beta\left(\frac{y-\mu}{\sigma}\right)^{3}\right)^{2}+1}{2+\alpha^{2} \frac{\pi^{2}}{3}+\frac{31}{21} \beta^{2} \pi^{6}+\frac{14}{15} \alpha \beta \pi^{4}} \times \frac{e^{-\left(\frac{y-\mu}{\sigma}\right)}}{\left(1-e^{-\left(\frac{y-\mu}{\sigma}\right)}\right)^{2}} .
$$

This fact is denoted by $Y \sim A B S L G(\alpha, \beta, \mu, \sigma)$.

\subsection{Method of Moments}

Let $m_{1}, m_{2}, m_{3}$ are respectively the first, three sample raw moments for a given random sample $z_{1}, z_{2}, \ldots, z_{n}$ of size $n$ drawn from $Z \sim A B S L G(\alpha, \beta, \mu, \sigma)$ distribution. Then the moment estimates of the four parameters $\alpha, \beta, \mu, \sigma$ are obtained by simulataneously solving the following equations derived by equating first three population moments with corresponding sample moments.

$$
m_{1}=\mu-\frac{\sigma}{2+\alpha^{2} \frac{\pi^{2}}{3}+\frac{31}{21} \beta^{2} \pi^{6}+\frac{14}{15} \alpha \beta \pi^{4}} \times\left(\frac{-5 \alpha \pi^{2}-\beta \pi^{4}}{15}\right),
$$

So,

$$
\begin{aligned}
\mu=m_{1} & +\frac{\sigma}{2+\alpha^{2} \frac{\pi^{2}}{3}+\frac{31}{21} \beta^{2} \pi^{6}+\frac{14}{15} \alpha \beta \pi^{4}} \times\left(\frac{-5 \alpha \pi^{2}-\beta \pi^{4}}{15}\right) \\
m_{2}= & \mu^{2}+\frac{2 \sigma \mu}{2+\alpha^{2} \frac{\pi^{2}}{3}+\frac{31}{21} \beta^{2} \pi^{6}+\frac{14}{15} \alpha \beta \pi^{4}} \times\left(\frac{-5 \alpha \pi^{2}-\beta \pi^{4}}{15}\right) \\
& +\sigma^{2} \frac{1}{2+\alpha^{2} \frac{\pi^{2}}{3}+\frac{31}{21} \beta^{2} \pi^{6}+\frac{14}{15} \alpha \beta \pi^{4}} \\
& \times\left(\frac{70 \pi^{2}+7 \alpha^{2} \pi^{4}+465 \alpha \beta \pi^{6}+896 \beta^{2} \pi^{8}}{210}\right)
\end{aligned}
$$

Let $E(Z)=a$ and $E\left(Z^{2}\right)=b$, then substituting the value of $\mu$ in equation (7),

$$
m_{2}=\left(m_{1}+a \sigma\right)^{2}-2 \sigma a\left(m_{1}+a \sigma\right)+\sigma^{2} b,
$$

So,

$$
\begin{gathered}
\sigma^{2}=\frac{m_{2}-m_{1}^{2}}{b-a^{2}} . \\
m_{3}=\mu^{3}+\frac{2 \sigma \mu^{2}}{2+\alpha^{2} \frac{\pi^{2}}{3}+\frac{31}{21} \beta^{2} \pi^{6}+\frac{14}{15} \alpha \beta \pi^{4}} \times\left(\frac{-5 \alpha \pi^{2}-\beta \pi^{4}}{15}\right) \\
+2 \mu \sigma^{2} \frac{1}{2+\alpha^{2} \frac{\pi^{2}}{3}+\frac{31}{21} \beta^{2} \pi^{6}+\frac{14}{15} \alpha \beta \pi^{4}} \\
\times\left(\frac{70 \pi^{2}+7 \alpha^{2} \pi^{4}+465 \alpha \beta \pi^{6}+896 \beta^{2} \pi^{8}}{210}\right) \\
+\frac{\sigma^{3}\left(-49 \alpha \pi^{4}-155 \beta \pi^{6}\right)}{105\left(2+\alpha^{2} \frac{\pi^{2}}{3}+\frac{31}{21} \beta^{2} \pi^{6}+\frac{14}{15} \alpha \beta \pi^{4}\right)} .
\end{gathered}
$$

while the exact solution of the equation (8) is not tractable, it can be numerically solved to estimate $\alpha, \beta$. 


\subsection{Maximum Likelihood Estimation}

Let $Y=\left(Y_{1}, Y_{2}, \ldots, Y_{n}\right)$ denote a random sample of size $n$ drawn from the $A B S L G(\alpha, \beta, \mu, \sigma)$ distribution. From the equation (6) the likelihood function is given by,

$$
\begin{aligned}
l & =\ln L(\alpha, \beta, \mu, \sigma ; Y)=-n \ln \left(2+\alpha^{2} \frac{\pi^{2}}{3}+\frac{31}{21} \beta^{2} \pi^{6}+\frac{14}{15} \alpha \beta \pi^{4}\right) \\
& +\sum_{i=1}^{n}\left[\log \left(\left(1-\alpha\left(\frac{y_{i}-\mu}{\sigma}\right)-\beta\left(\frac{y_{i}-\mu}{\sigma}\right)^{3}\right)^{2}+1\right)\right. \\
& \left.-2 \log \left(1+e^{-\left(\frac{y_{i}-\mu}{\sigma}\right)}\right)\right]-\sum_{i=1}^{n}\left(\frac{y_{i}-\mu}{\sigma}\right) .
\end{aligned}
$$

The MLE of $\alpha, \beta, \mu$ and $\sigma$ are obtained by numerically maximizing $\log \mathrm{L}$ with respect to $\alpha, \beta, \mu$ and $\sigma$. The variance-covariance matrix of the MLEs can be derived by using the asymptotic distribution of MLEs as,

$$
\left[\begin{array}{llll}
E\left(\frac{-\partial^{2} l}{\partial \alpha^{2}}\right) & E\left(\frac{-\partial^{2} l}{\partial \alpha \partial \beta}\right) & E\left(\frac{-\partial^{2} l}{\partial \alpha \partial \mu}\right) & E\left(\frac{-\partial^{2} l}{\partial \alpha \partial \sigma}\right) \\
E\left(\frac{-\partial^{2} l}{\partial \beta \partial \alpha}\right) & E\left(\frac{-\partial^{2} l}{\partial \beta^{2}}\right) & E\left(\frac{-\partial^{2} l}{\partial \beta \partial \mu}\right) & E\left(\frac{-\partial^{2} l}{\partial \partial \partial \sigma}\right) \\
E\left(\frac{-\partial^{2} l}{\partial \mu \partial \alpha}\right) & E\left(\frac{-\partial^{2} l}{\partial \mu \partial \beta}\right) & E\left(\frac{-\partial^{2} l}{\partial \mu^{2}}\right) & E\left(\frac{-\partial^{2} l}{\partial \mu \partial \sigma}\right) \\
E\left(\frac{-\partial^{2} l}{\partial \sigma \partial \alpha}\right) & E\left(\frac{-\partial^{2} l}{\partial \sigma \partial \beta}\right) & E\left(\frac{-\partial^{2} l}{\partial \sigma \partial \mu}\right) & E\left(\frac{-\partial^{2} l}{\partial \sigma^{2}}\right)
\end{array}\right]^{-1}
$$

When the exact expressions for various expectations above are cumbersome, in practice they are estimated as

$$
E\left(\frac{-\partial^{2} l}{\partial \alpha^{2}}\right) \approx \frac{-\partial^{2} l}{\partial \alpha^{2}}\left|\alpha=\widehat{\alpha}, E\left(\frac{-\partial^{2} l}{\partial \alpha \partial \beta}\right) \approx \frac{-\partial^{2} l}{\partial \alpha \partial \beta}\right| \alpha=\widehat{\alpha}, \beta=\widehat{\beta}, \text { etc. }
$$

\section{Simulation}

In this section, we conduct the Monte Carlo simulation studies to assess on the finite sample behavior of the MLEs of $\alpha, \beta, \mu$ and $\sigma$. All results are obtained from 5000 Monte Carlo replications and the simulations were carried out using the package 'maxLik' in statistical software R. In each replication, a random sample of size $n$ is drawn from the $A B S L G(\alpha, \beta, \mu, \sigma)$ distribution.

The true parameter values used in the data generating processes are $\mu=0$, and $\sigma=1$ and different values for the parameters $\alpha=(-0.5,0,0.5,1)$ and $\beta=(-0.5,0.0,0.5,1)$. Tables 1 represent the empirical means and the average mean squared errors (AMSE) of the corresponding estimators for sample sizes $n=25,50,100,200$ and 400. It is noticeable that the estimation of the parameters $\alpha$ and $\beta$ are the most hard situations, whereas the estimation of $\mu$ and $\sigma$ are the most tractable.

We note that in all cases the biases and AMSEs of the MLEs of $\alpha, \beta, \mu$ and $\sigma$ decay toward zero when the sample size increases, as expected. There is a small sample bias in the estimates of the model parameters.

Figures 4 and 5 represents the AMSE of the parameters $\alpha$ and $\beta$ for fixed values of another parameters in the model. As can be seen in the Figures, by increasing the sample size, the AMSE of estimated parameters decreases.

\section{Real Data}

To illustrate the applicability of the proposed model in section 2, three real data sets are analysed. Throughout this section, the statistical software $\mathrm{R}$ with the package DEoptim was used for maximizing the corresponding liklihood functions. We compared the proposed distribution $A B S L G(\alpha, \beta, \mu, \sigma)$ with the logistic distribution 
Table 1. Monte Carlo simulation results: The average mean square error of $\alpha, \beta, \sigma$, and $\mu$.

\begin{tabular}{|c|c|c|c|c|c|c|c|c|c|c|c|c|}
\hline $\mathrm{n}$ & \multicolumn{4}{|c|}{$\alpha=-0.5, \beta=-0.5$} & \multicolumn{4}{|c|}{$\alpha=0.0, \beta=-0.5$} & \multicolumn{4}{|c|}{$\alpha=0.5, \beta=-0.5$} \\
\hline & & $\beta$ & $\mu$ & $\bar{\sigma}$ & 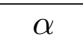 & $\bar{\beta}$ & $\mu$ & $\bar{\sigma}$ & & $\beta$ & $\mu$ & $\bar{\sigma}$ \\
\hline 25 & & .72 & 1. & & & 0.4 & & & & 0.72 & & \\
\hline 50 & & & & & & & & & & & & \\
\hline & & & & & & & & & & & 91 & \\
\hline & & 0. & & & & & & & & 0.63 & .74 & \\
\hline 400 & 39 & 0.58 & 0.8 & 0.3 & & 0.20 & 0.38 & & 31 & 0.47 & 0.65 & U. \\
\hline $\mathrm{n}$ & \multicolumn{4}{|c|}{$\alpha=-0.5, \beta=0.0$} & \multicolumn{4}{|c|}{$\alpha=0.0, \beta=0.0$} & \multicolumn{4}{|c|}{$\alpha=0.5, \beta=0.0$} \\
\hline & & $\beta$ & $\sigma$ & $\mu$ & $\alpha$ & $\beta$ & $\sigma$ & $\mu$ & $\alpha$ & $\beta$ & $\sigma$ & $\mu$ \\
\hline 25 & & 0.46 & & 0.8 & & 0.27 & 0.92 & & 1 & 0.40 & 1.19 & 0.65 \\
\hline 5 & & & & & & 0.25 & & & 77 & 0.31 & 0.70 & 0.61 \\
\hline & & & & & & 0. & 0 & & 8 & j & 0 & 0.54 \\
\hline & 9 & & & & & & 0.6 & & & 0. & 0. & 0. \\
\hline 400 & 65 & 0.17 & 0.53 & 0.3 & 17 & 0.1 & 0.21 & & .47 & 0.21 & 0.41 & 0. \\
\hline $\mathrm{n}$ & \multicolumn{8}{|c|}{$\alpha=-0.5, \beta=0.5$} & \multicolumn{4}{|c|}{$k=0.5, \beta=0$} \\
\hline & & $\beta$ & & & & $\beta$ & $O$ & & & $\beta$ & $\sigma$ & $\mu$ \\
\hline 2 & & & & & & & & & & & & .85 \\
\hline 50 & & 0.37 & & & & & & & 15 & 0.24 & .05 & 0.36 \\
\hline 10 & 9 & 0.35 & & 0. & & 0.18 & 0.83 & & 13 & 0.17 & 0.78 & 0.32 \\
\hline 20 & 58 & 0.33 & 0.7 & 0.35 & & 0.14 & 0.46 & 0. & 0.12 & 0.14 & 0.55 & 0.31 \\
\hline 400 & .59 & 0.26 & 0.48 & 0.27 & 0.14 & 0.11 & 0.31 & 0.21 & 0.08 & 0.11 & 0.30 & 0.27 \\
\hline
\end{tabular}

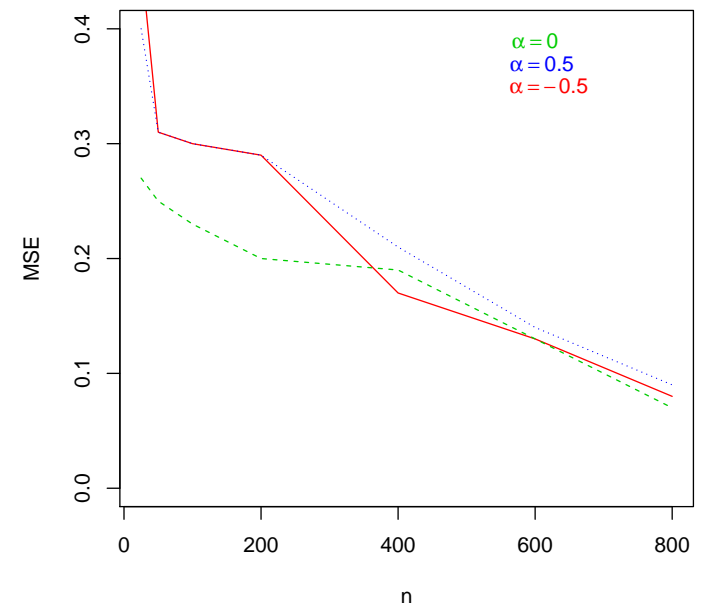

Figure 4. The mean square error of parameter $\beta$ when the true value is zero for different values of parameter $\alpha$.

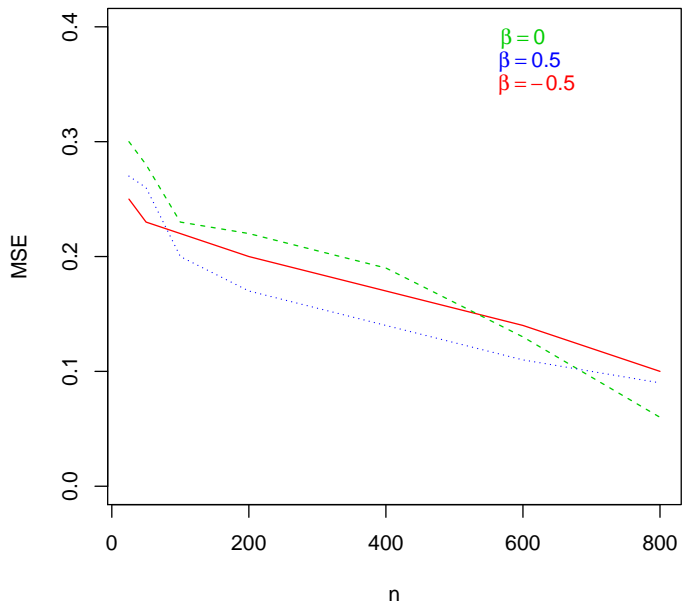

Figure 5. The mean square error of parameter $\alpha$ when the true value is zero for different values of parameter $\beta$.

$L G(\mu, \sigma)$, skew logistic distribution $(\lambda, \mu, \sigma)$ of [13], the new skew logistic distribution $S L(\lambda, \alpha, \mu, \sigma)$ of [4], alpha-skew-normal distribution $A S N(\alpha, \beta, \mu)$ of [7], alpha-skew-Laplace distribution $A S L(\alpha, \beta, \mu)$ of Harandi and Alamatsaz (2013), alpha skew normal slash distribution $A S N S(\alpha, q, \mu, \sigma)$ of [8], and alpha skew logistic 


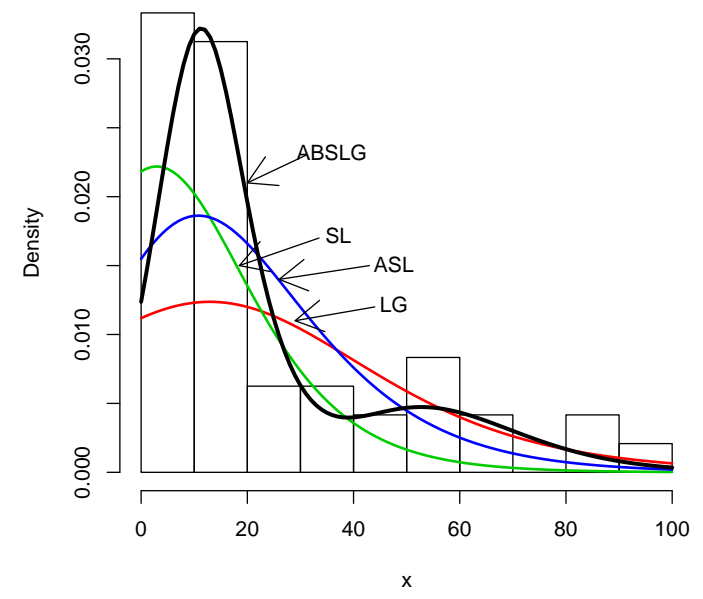

Figure 6. Plots of expected densities and ABSLG for application 1

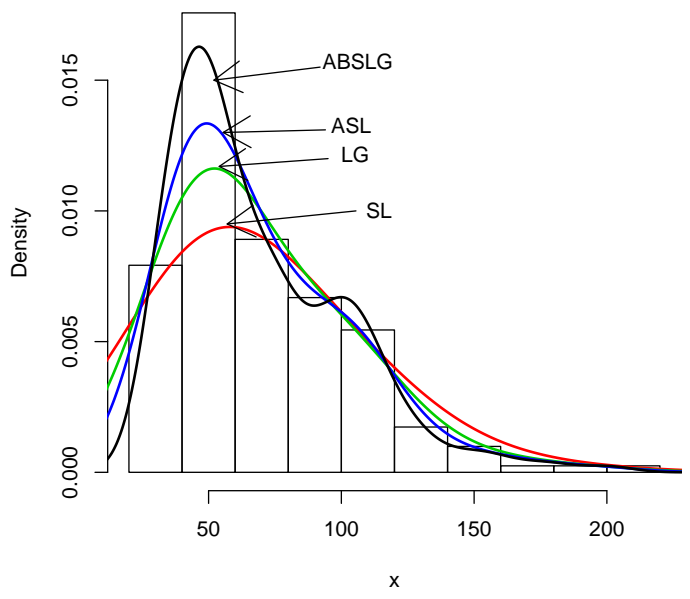

Figure 7. Plots of expected densities and ABSLG for application 2

distribution $A S L G(\alpha, \mu, \sigma)$ of Hazarika and [5]. Akaike Information Criterion (AIC) and Beysian Information Criterion (BIC) are used for model comparison.

Further, since are nested models the likelihood ratio (LR) test is used to discriminate between them. The LR test is carried out to test the following hypothesis:

$H_{0}: \alpha=\beta=0$, that is the sample is drawn from $L G(\mu, \sigma)$

against the alternative

$H_{1}: \alpha \neq 0, \beta \neq 0$, that is the sample is drawn from $A B S L G(\alpha, \beta, \mu, \sigma)$.

Aplication 6.1. For illustration purposes, we consider the uncensored part of a data set analysed by Leiva et al. (2007) corresponding to the survival times ( $\mathrm{T}$, in months) of 48 patients who were treated with alkylating agents for multiple myeloma. These data (which we will henceforth call myeloma) are :1, 1, 2, 2, 2, 3, 5, 5, 6, 6, 6, 6, 7, $7,7,9,11,11,11,11,11,13,14,15,16,16,17,17,18,19,19,24,25,26,32,35,37,41,42,51,52,54,58,66$, $67,88,89,92$. By comparing the AICs and BICs, one can see the ABSLG distribution is the best among the fitted models.

LR test result: the value of LR test statistics is 21.92 which exceed the $95 \%$ critical value. Thus there is evidence in favors of the alternative hypothesis that the sampled data comes from $A B S L G(\alpha, \beta, \mu, \sigma)$ not from $A S L G(\alpha, \mu, \sigma)$. The values of these statistics for the models are listed in Table 2. Overall,by comparing the measures of these formal goodness-of- tests in Table 2, we conclude that the ABSLG distribution yields a better than the LG and ASLG distributions and therefore it can be an interesting alternative to these distributions for modeling lifetime data. These results illustrate the importance of the additional shape parameters of the new distribution to analyze real data.

Aplication 6.2. The second data $\operatorname{set}(\mathrm{n}=202)$ in Table 3 is from Weisberg (2005) and it represents the sum of skin folds in 202 athletes collected at the Australian Institute of Sport.The data sets:28.0, 109.1, 102.8, 104.6, 126.4, 80.3, 75.2, 87.2, 97.9, 75.1, 65.1, 171.1,76.8, 117.8, 90.2, 97.2, 99.9, 125.9, 69.9, 98, 96.8, 80.3, 74.9, 83.0, 91.0, 76.2, 52.6, 111.1,110.7, 74.7, 113.5, 99.8, 80.3, 109.5, 123.6, 91.2, 49.0, 110.2, 89.0, 98.3, $122.1,90.4,106.9,156.6,101.1,126.4,114.0,70.0,77.0,148.9,80.1,156.6,115.9,181.7,71.6,143.5,200.8$, 68.9,103.6, 71.3, 54.6, 88.2, 95.4, 47.5, 55.6, 62.9, 52.5, 62.6, 49.9,57.9, 109.6, 98.5, 136.3, 103.6,102.8, 131.9, $33.8,43.5,46.2,73.9,36.8,67,41.1,59.4,48.4,50.0,54.6,42.3,46.1,46.3,109.0,98.1,80.6,68.3,47.6,61.9$, $38.2,43.5,56.8,41.6,58.9,44.5,41.8,33.7,50.9,40.5,51.2,54.4,52.3,57.0,65.3,52.0,42.7,35.2,49.2,61.8$, 
Table 2. Estimates of the parameters, log likelihood, AIC and BIC for fitted model for real data.

\begin{tabular}{|c|cccccccc|}
\hline & $\alpha$ & $\beta$ & $\mu$ & $\sigma$ & $\lambda$ & $\log$-liklihood & AIC & BIC \\
\hline$L G(\mu, \sigma)$ & - & - & 20.21 & 12.93 & - & -219.97 & 443.95 & 447.69 \\
\hline$L_{s}(\lambda, \alpha, \mu, \sigma)$ & -0.20 & - & 13.35 & 10.82 & -0.50 & -217.68 & 443.37 & 450.85 \\
\hline$A S N(\alpha, \beta, \mu)$ & 0.26 & 0.042 & 11.45 & - & - & -218.41 & 442.82 & 448.44 \\
\hline$A S L(\alpha, \beta, \mu)$ & -0.43 & 0.015 & 12.37 & - & - & -217.96 & 441.93 & 447.54 \\
\hline$A S L G(\alpha, \mu, \sigma)$ & -0.30 & - & 10.26 & 11.38 & - & -217.16 & 440.32 & 445.94 \\
\hline$A B S L G(\alpha, \beta, \mu, \sigma)$ & 0.31 & -0.03 & 14.86 & 6.18 & - & $\mathbf{- 2 0 9 . 0 2}$ & $\mathbf{4 2 6 . 0 4}$ & $\mathbf{4 3 3 . 5 2}$ \\
\hline
\end{tabular}

Table 3. LR test

\begin{tabular}{|c|c|c|c|}
\hline Aarset & Hypotheses & Statistic & P-Value \\
\hline ABSLG vs LG & $H_{0}: \alpha=\beta=0$ vs $H_{1}: H_{0}$ is false & 21.92 & $<0.0001$ \\
\hline ABSLG vs ASLG & $H_{0}: \beta=0$ vs $H_{1}: H_{0}$ is false & 16.28 & 0.0011 \\
\hline
\end{tabular}

$46.5,34.8,60.2,48.1,44.5,54.0,44.7,64.9,43.8,58.3,52.8,43.1,78.0,40.8,41.5,50.9,49.6,88.9,48.3,61.8$, 43.0, 61.1, 43.8, 54.2, 41.8, 34.1,30.5, 34.0, 46.7, 71.1, 65.9, 34.3, 34.6, 31.8, 34.5, 31.0, 32.6, 31.5, 32.6, 31.0, $33.7,30.3,38.0,55.7,37.5,112.5,82.7,29.7,38.9,44.8,30.9,44.0,37.5,37.6,31.7,36.6,48,41.9,30.9,52.8,43.2$, $113.5,96.9,49.3,42.3,96.3,56.5,105.7,100.7,56.8,75.9,52.8,47.8,76.0,61.2,75.6,43.3,49.5,70.0,75.7,57.7$, $67.2,56.5,47.6,60.4,34.9$.

LR test result: The value of LR test statistics is 23.61 which exceed the $95 \%$ critical value. Thus, there is evidence in favors of the alternative hypothesis that the sampled data comes from $A B S L G(\alpha, \beta, \mu, \sigma)$ not from $A S L G(\alpha, \mu, \sigma)$.

Table 4. Estimates of the parameters, log likelihood, AIC and BIC for fitted model for real data.

\begin{tabular}{|c|cccccccc|}
\hline & $\alpha$ & $\beta$ & $\mu$ & $\sigma$ & $\lambda$ & $\log$-liklihood & AIC & BIC \\
\hline$L G(\mu, \sigma)$ & - & - & 65.17 & 17.98 & - & -986.84 & 1977.69 & 1984.31 \\
\hline$L_{s}(\lambda, \alpha, \mu, \sigma)$ & -0.31 & - & 51.33 & 16.56 & -1.54 & -982.29 & 1972.59 & 1980.07 \\
\hline$A S N(\alpha, \beta, \mu)$ & 0.42 & 0.37 & 49.98 & - & - & -981.92 & 1969.85 & 1979.34 \\
\hline$A S L(\alpha, \beta, \mu)$ & -0.24 & 0.10 & 48.73 & - & - & -980.48 & 1966.97 & 1976.46 \\
\hline$A S L G(\alpha, \mu, \sigma)$ & -0.27 & - & 51.91 & 16.45 & - & -979.84 & 1965.68 & 1975.61 \\
\hline$A B S L G(\alpha, \beta, \mu, \sigma)$ & 0.31 & -0.027 & 14.86 & 6.18 & - & $\mathbf{- 9 7 5 . 0 4}$ & $\mathbf{1 9 5 8 . 0 8}$ & $\mathbf{1 9 7 1 . 3 2}$ \\
\hline
\end{tabular}

\section{Conclusion}

In this paper, we proposed a new family of distributions with two extra generator parameters, which includes as special cases of logistic, ASLG, BSLG and BLG distributions and some of its basic properties are investigated which include moments, Skewness and Kurtosis functions, mean deviations. Also the below zero truncated version of the proposed distribution was presented as a potential life time distribution. The application of the new family is straightforward. The model parameters are estimated by maximum likelihood and two real examples are used for illustration, where the new family does fit well both data sets.

Table 5. LR test

\begin{tabular}{|c|c|c|c|}
\hline Aarset & Hypotheses & Statistic & P-Value \\
\hline ABSLG vs LG & $H_{0}: \alpha=\beta=0$ vs $H_{1}: H_{0}$ is false & 23.61 & $<0.0001$ \\
\hline ABSLG vs ASLG & $H_{0}: \beta=0$ vs $H_{1}: H_{0}$ is false & 9.61 & 0.01 \\
\hline
\end{tabular}




\section{REFERENCES}

1. M. Alizadeh, M. Emadi, M. Doostparast, A New Two-Parameter Lifetime Distribution: Properties, Applications and Different Method of Estimations, Statistics, Optimization and Information Computing, vol. 7(2), pp. 291-310, 2019.

2. A. Azzalini, Further results on a class of distributions which includes the normal ones, Statistics, vol. 46(2), pp. 199-208, 1986.

3. N. Balakrishnan, Handbook of the logistic distribution, Inc., New York, Basel, 1992.

4. S. Chakraborty, Hazarika, and M. M. Ali, A new skew logistic distribution and its properties, Pakistan Journal of Statistics, vol. 28(8), pp. 513-524, 2012.

5. S. Chakraborty, Hazarika, and M. M. Ali, A multimodal skew-laplace distribution: its properties and applications, Pakistan Journal of Statistics, vol. 30(2), pp. 253-264, 2014.

6. S. Chakraborty, Hazarika, and M. M. Ali, A multimodal skewed extension of normal distribution: its properties and applications, Statistics, vol. 49(4), pp. 859-877, 2015

7. D. Elal-Olivero, H. W. Gómez, and F. A. Quintana, Bayesian modeling using a class of bimodal skew-elliptical distributions, Journal of Statistical Planning and Inference, vol. 139(4), pp. 1484-1492, 2009.

8. W. Gui, A generalization of the slashed distribution via alpha skew normal distribution, Statistical Methods and Applications, vol. 23(4), pp. 547-563, 2014.

9. P. Hazarika, and S. Chakraborty, Alpha-skew-logistic distribution, IOSR Journal of Mathematics, vol. 10(4), pp. 36-46, 2014.

10. W. J. Huang, and Y.-H. Chen, Generalized skew-cauchy distribution, Statistics and probability letters, vol. 77(11), pp. 1137-1147, 2007.

11. M. Mozafari, M. Afshari, M. Alizadeh, and H. Karamikabir, The Zografos-Balakrishnan Odd Log-Logistic Generalized Half-Normal Distribution with Mathematical Properties and Simulations, Statistics, Optimization and Information Computing, vol. 7(1), pp. 211234, 2019.

12. S. Shafiei, M. Doostparast, and A. Jamalizadeh, The alpha-beta skew normal distribution: properties and applications, Statistics, vol. 50(2), pp. 338-349, 2016.

13. A. Wahed, and M. M. Ali, The skew-logistic distribution, Journal of Statistical Research, vol. 35(2), pp. 71-80, 2001. 